\title{
Leaf and fruit essential oil compositions of Pimenta guatemalensis (Myrtaceae) from Costa Rica
}

\author{
Carlos Chaverri \& José F. Cicció \\ Escuela de Química y Centro de Investigaciones en Productos Naturales (CIPRONA), Universidad de Costa Rica, \\ 11501-2060, San José, Costa Rica; carloschaverri@yahoo.com, jfciccio@gmail.com
}

\author{
Received 15-V-2014. Corrected 20-IX-2014. Accepted 22-X-2014.
}

\begin{abstract}
Pimenta is a genus of flowering plants in the Myrtaceae family, which has about 15 species, mostly found in the Caribbean region of the Americas. Commonly used for culinary and medicinal purposes, the best known commercial species are allspice, $P$. dioica ( $P$. officinalis) and bay rum, $P$. racemosa, but there is little information concerning $P$. guatemalensis. The aim of the present study was to identify the chemical composition of the leaf and fruit essential oils of $P$. guatemalensis. The extraction of essential oils of $P$. guatemalensis growing wild in Costa Rica was carried out by the hydrodistillation method at atmospheric pressure, using a modified Clevenger type apparatus. The chemical composition of the oils was analyzed by capillary gas chromatographyflame ionization detector (GC/FID) and gas chromatography-mass spectrometry (GC/MS) using the retention indices on DB-5 type capillary column. A total of 103 and 63 compounds were identified in the leaf and fruit oils, respectively, corresponding to $96.8 \%$ and $86.1 \%$ of the total amount of the oils. The leaf oil consisted mainly of eugenol (72.8\%), and mono- and sesquiterpene hydrocarbons (18.2\%). Among terpenes the major components were $\beta$-caryophyllene (8.2\%) and terpinolene (3.0\%). The fruit oil also consisted mainly of eugenol (74.7\%) and minor amounts of oxygenated mono- and sesquiterpenes $(7.3 \%)$, mainly caryophyllene oxide (3.3\%). This is the first report of the chemical composition of the essential oils obtained from this plant species. Rev. Biol. Trop. 63 (1): 303-311. Epub 2015 March 01.
\end{abstract}

Key words: Pimenta guatemalensis, Myrtaceae, essential oil composition, eugenol, $\beta$-caryophyllene, caryophyllene oxide, terpinolene, Costa Rica.

Myrtaceae is a family constituted of about 142 genera and includes more than 5500 species of mostly trees and shrubs with conspicuous oil glands, distributed predominantly in the Southern hemisphere, mainly in Australia and South America (Wilson, 2011).

Worldwide, the family has a considerable economic value because species from several genera are used as a source of timber (for example, Eucalyptus globulus Labill., Tasmanian blue gum). Also, some species provide edible fruits [Psidium guajava L., guava; $P$. friedrichsthalianum (O. Berg) Nied., cas and Eugenia uniflora L., pitanga], are utilized as spices [Syzygium aromaticum (L.) Merr. \& L. M. Perry, clove, and Pimenta dioica (L.) Merr., allspice], as a source of commercial essential oils [clove, Melaleuca alternifolia Cheel, teatree oil, Eucalyptus spp. and Pimenta racemosa (Mill.) J.W. Moore, bay rum oil] and many Myrtaceae are important in horticulture as flowering ornamentals (Callistemon spp., Melaleuca spp.).

The genus Pimenta Lindl. contains about 15 species, mostly in the Caribbean region of the Americas. Pimenta guatemalensis (Lundell) Lundell is a tree about 5-16(20) $\mathrm{m}$ tall with a straight trunk and reddish brown bark (Barrie, 2007). In Costa Rica, it is commonly known as Jamaica (León, \& Poveda, 2000). This plant is distributed along Central America, from Guatemala to Panama. The young branchlets are 4-angled. The leaves are evergreen, simple, opposite and oblanceolate to elliptical, 
the blade cuneate at the base and acuminate at the apex. When the leaves are crushed they give off a scent with an aromatic flavor resembling a mixture of clove and cinnamon due to an oil of unknown composition. The fragrant flowers are small and the receptacle has five calyx lobes, arranged in panicles ( $c a .20$ flowers) in the leaf axils. The ovaries are uni-locular or without loculi. This plant grows wild in Costa Rica and it can be found distributed between 250 and $900 \mathrm{~m}$ above sea level mostly in the rain forests of the North region and Caribbean slopes. To the best of our knowledge, no previous reports on the chemistry of $P$. guatemalensis have been published.

Pimenta species are used in several countries of Mesoamerica and Caribbean for culinary and medicinal purposes (Morton, 1981; Germosén-Robineau, 2005). Paula, Reis, Ferreira, Menezes, and Paula (2010), and Rao, Navinchandra, and Jayaveera (2012) and Lim (2012) have recently reviewed some botanical aspects, traditional medicinal uses, biological and pharmacological activities and chemical compositions of Pimenta dioica and P. racemosa (the most important from the economical point of view) and P. pseudocaryophyllus, the only species of this genus that grows in the Brazilian Atlantic Rain Forest in South America (Marques et al., 2010; Paula et al., 2011). Formerly the name Pimenta dioica was frequently misapplied to $P$. guatemalensis native populations in Costa Rica (Barrie, 2007).

The chemical composition of Pimenta spp. leaf oils produced in different countries has been investigated thoroughly (Tucker, Maciarello, \& Landrum, 1991a; Tucker, Maciarello, Adams, Landrum, \& Zanoni, 1991b; Tucker, Maciarello, Adams, Landrum, \& Zanoni, 1991c; Tucker, Maciarello, \& Landrum, 1992a; Tucker, Maciarello, \& Landrum, 1992b; Bello et al., 1995; Ayedoun et al., 1996; Pino, \& Rosado, 1996; Bello, Urquiola, García, Rosado, \& Pino, 1998; Pino, Bello, \& Urquiola, 2002; Jirovetz et al., 2007). The major component of the leaf oil of $P$. dioica from Jamaica and Cuba was the phenylpropanoid eugenol ranging from 54.3 to $79.2 \%$ with lesser amounts of the monoterpenoid 1,8-cineole (1.1-4.6\%) and the sesquiterpenoids $\beta$-caryophyllene (1-8.7\%), $\alpha$-humulene (1.0-3.9\%) and caryophyllene oxide (0.2-1.8\%) (Tucker et al., 1991a; Pino, \& Rosado, 1996). The foliar oil of P. haitiensis (Urb.) L. R. Landrum of the Dominican Republic, presented high amounts of the phenylpropanoids methyl chavicol (11.7-41.1\%) or methyl eugenol (0.6$24.4 \%)$ and trans-anethole (4.7-8.7\%), and the monoterpenoids 1,8-cineole (11.4-33.1\%), linalool (16.0-17.8\%) and $\alpha$-terpineol (2.85.6\%) (Tucker et al., 1991c). Pimenta jamaicensis (Britton, \& Harris) Proctor of Jamaica gave leaf oils which were dominated by either eugenol (61.8\%), limonene (10.4\%) and $\beta$-caryophyllene $(5.8 \%)$, or 1,8 -cineole (43.4$43.9 \%), \alpha$-terpineol (0.3-18.0\%), terpinen-4-ol (6.4-7.2\%) and $p$-cymene (2.2-10.4\%) (Tucker et al., 1992a). Sesquiterpenoids dominated the oil of one collection of P. obscura Proctor of Jamaica (51.8\%) with ledol (13.5\%) and palustrol $(7.6 \%)$ as main constituents (Tucker et al., $1992 \mathrm{~b})$. Other samples were found to be rich in 1,8-cineole (16.8-25.1\%), limonene (5.3$11.5 \%), p$-cymene (ca. 11\%), $\alpha$-terpineol (6.7$8.1 \%$ ) and terpinen-4-ol (4.9-9.8\%) (Tucker et al., 1992a). Tucker et al. (1991b) studied the foliar oils of three varieties of $P$. racemosa and two commercial bay oils. The leaf oil of commercial variety was composed mainly of phenylpropanoids [eugenol (44.4-68.9\%), chavicol (traces-15.5\%) and/or methyl eugenol $(11.9 \%)]$, together with variable amounts of myrcene (0.1-16.2\%). This composition is similar to the same variety cultivated in Benin (Ayedoun et al., 1996) and North India (Pragadheesh, Yadav, Singh, Gupta, \& Chanotiya, 2013). From this study the authors were able to ascertain that leaf oils of varieties of $P$. racemosa may be dominated by chavicol, 1,8-cineole, eugenol, methyl chavicol, geraniol, limonene, methyl eugenol, myrcene, trans-methyl isoeugenol, $\gamma$-terpinene, terpinen4-ol, $\alpha$-terpineol and/or thymol (Tucker et al., 1991b; Bello et al., 1995). The leaf oil from $P$. adenoclada (Urb.) Burret from Cuba (Pino et al., 2002) was dominated by the oxygenated sesquiterpenes caryophyllene oxide (15.4\%), 
$\alpha$-muurolol (9.4\%) and humulene epoxide II (7.6\%). Pimenta pseudocaryophyllus (Gomes) L. R. Landrum, native to Brazil, gave leaf oils dominated by chavibetol $(70.9 \%)$ and methyl eugenol (20.7\%) (Marques et al., 2010). A recent study of this species in central Brazilian Cerrado (Paula et al., 2011) showed three chemotypes: one composed mainly by citral [geranial (ca. 40\%) and neral (ca. 25\%)], a second type with high amount of asarone (ca. 65\%) and another with high amounts of $(E)$-methyl isoeugenol (ca. 93\%).

The chemical composition of $P$. dioica fruit (pimento-allspice) oil has been the subject of several studies. One review paper reported, that this oil contains eugenol (68.6-87.0\%), methyl eugenol (2.9-13.0\%), $\beta$-caryophyllene (2.5-5.4\%) and 1,8-cineole (2.3-3.3\%) as main constituents (Pino, 1999, and references therein cited). The oils from berries collected in Xaltipan, Puebla, Mexico (García Fajardo et al., 1997), and extracted by steam distillation and hydrodistillation, were composed mainly by methyl eugenol (48.3-62.7\%), myrcene (16.5$17.7 \%)$ and, eugenol (8.3-17.3\%).

In the present work we report the chemical composition of the oils isolated by hydrodistillation from leaves and fruits of P. guatemalensis collected in Costa Rica.

\section{MATERIALS AND METHODS}

Plant material: Leaves and fruits of Pimenta guatemalensis (Lundell) Lundell, Myrtaceae, growing wild in Costa Rica in a rain forest, were collected in August 2005 (rainy season), in Bajo Rodríguez, San Ramón, Province Alajuela. A voucher specimen was kept at the Herbarium of the University of Costa Rica (USJ 77482).

Isolation of the essential oils: Air-dried (in the shade at room temperature) leaves (500g) of P. guatemalensis were subjected to hydrodistillation at atmospheric pressure, for three hours, using a modified Clevenger-type glass apparatus. The light yellowish distilled oil (2.5mL) was dried over anhydrous $\mathrm{Na}_{2} \mathrm{SO}_{4}$; and the yield $(\mathrm{v} / \mathrm{m})$ of the oil was $0.5 \%$. The same procedure was utilized for the air-dried fruits $(250 \mathrm{~g})$. The colorless oil was dried, yielding $1.8 \mathrm{~mL}(0.7 \% \mathrm{v} / \mathrm{m})$. The oils were filtered and stored at $0-10^{\circ} \mathrm{C}$ in the dark for further analysis.

GC/FID analysis: The oils of $P$. guatemalensis were analyzed by GC-FID (gas chromatography with flame ionization detector) using a Shimadzu GC-2014 gas chromatograph. The data were obtained on a poly $(5 \%$ phenyl-95\% methylsiloxane) fused silica capillary column $(30 \mathrm{~m} \times 0.25 \mathrm{~mm}$; film thickness $0.25 \mu \mathrm{m})$, (MDN-5S, Supelco), with a LabSolutions, Shimadzu GC Solution, Chromatography Data System, software version 2.3. Operating conditions were: carrier gas $\mathrm{N}_{2}$, flow $1.0 \mathrm{~mL} /$ min; oven temperature program: $60-280^{\circ} \mathrm{C}$ at $3^{\circ} \mathrm{C} / \mathrm{min}, 280^{\circ} \mathrm{C}(2 \mathrm{~min})$; sample injection port temperature $250^{\circ} \mathrm{C}$; detector temperature $280^{\circ} \mathrm{C}$; split 1:60.

GC/MS analysis: The analyses by gas chromatography coupled to mass selective detector were performed using a Shimadzu GC-17A gas chromatograph coupled with a GCMS-QP5000 apparatus and CLASS 5000 software with Wiley 139 and NIST computer databases. The data were obtained using the same column described above. Operating conditions were: carrier gas $\mathrm{He}$, flow $1.0 \mathrm{~mL} / \mathrm{min}$; oven temperature program: $60-280^{\circ} \mathrm{C}$ at $3{ }^{\circ} \mathrm{C} /$ min; sample injection port temperature $250^{\circ} \mathrm{C}$; detector temperature $260^{\circ} \mathrm{C}$; ionization voltage: $70 \mathrm{eV}$; ionization current $60 \mu \mathrm{A}$; scanning speed 0.5 s over $38-400 \mathrm{amu}$ range; split 1:70.

\section{Identification of chemical constituents:}

The oil components were identified using the retention indices (RI) on capillary DB-5 type column (van den Dool, \& Kratz, 1963), and by comparison of their mass spectra with those published in the literature (Adams, 2007) or those of the author's database. To obtain the retention indices for each peak, $0.1 \mu \mathrm{L}$ of $n$-alkane mixture (Sigma retention index standard for gas chromatography, $\mathrm{C}_{8}-\mathrm{C}_{32}, \mathrm{R} 8769$, 
USA) was injected under the same experimental conditions reported above. Integration of the total chromatogram (GC/FID), expressed as area percent, has been used to obtain quantitative compositional data.

\section{RESULTS}

From the hydrodistilled oils, a total of 126 compounds were identified using GC/FID and GC/MS, accounting for $96.8 \%$ (leaves) and $86.1 \%$ (fruits) of the total composition of the essential oils. The compounds identified in the leaf and fruit oils of $P$. guatemalensis are presented in Table 1, where the components were listed in order of elution on a MDN-5S column. Table 1 also includes the relative percentages of single components, their experimental retention indices (RI) with reference to a homologous series of linear alkanes $\left(\mathrm{C}_{8^{-}}\right.$ $\mathrm{C}_{32}$ ) and, for comparison purposes, previously published values. Additionally, percentages of oils various types/classes of constituents were also indicated.

TABLE 1

Percentage composition of the leaf and fruit oils of Pimenta guatemalensis from Costa Rica

\begin{tabular}{|c|c|c|c|c|c|}
\hline Compound $^{\mathrm{a}}$ & R.I. ${ }^{b}$ & R.I. ${ }^{\mathrm{c}}$ & Leaf & Fruit & Identification Method $^{\mathrm{d}}$ \\
\hline Pentan-1-ol & 762 & 762 & $\mathrm{t}$ & nd & 1,2 \\
\hline Hexanal & 801 & 801 & $\mathrm{t}$ & nd & 1,2 \\
\hline (2E)-Hexenal & 849 & 846 & 0.1 & $\mathrm{t}$ & 1,2 \\
\hline (2E)-Hexenol & 853 & 854 & $\mathrm{t}$ & nd & 1,2 \\
\hline Hexan-1-ol & 863 & 863 & $\mathrm{t}$ & $\mathrm{t}$ & 1,2 \\
\hline Heptanal & 901 & 901 & $\mathrm{t}$ & nd & 1,2 \\
\hline$(2 E, 4 E)$-Hexadienal & 907 & 907 & $\mathrm{t}$ & nd & 1,2 \\
\hline (3Z)-Hexenyl formate & 915 & 913 & nd & $\mathrm{t}$ & 1,2 \\
\hline$\alpha$-Thujene & 927 & 924 & 0.2 & $\mathrm{t}$ & 1,2 \\
\hline$\alpha$-Pinene & 933 & 932 & 0.1 & $\mathrm{t}$ & $1,2,3$ \\
\hline (2E)-Heptenal & 959 & 947 & $\mathrm{t}$ & nd & 1,2 \\
\hline Heptan-1-ol & 966 & 959 & 0.1 & $\mathrm{t}$ & 1,2 \\
\hline Hexanoic acid & 967 & 967 & $\mathrm{t}$ & nd & 1,2 \\
\hline$\beta$-Pinene & 978 & 974 & $\mathrm{t}$ & nd & $1,2,3$ \\
\hline 6-Methyl-5-hepten-2-one & 984 & 981 & $\mathrm{t}$ & nd & 1,2 \\
\hline Myrcene & 988 & 988 & 0.6 & 0.8 & 1,2 \\
\hline Butyl butanoate & 992 & 993 & $\mathrm{t}$ & nd & 1,2 \\
\hline Octanal & 1004 & 998 & 0.1 & nd & 1,2 \\
\hline$\alpha$-Phellandrene & 1008 & 1002 & 0.6 & nd & 1,2 \\
\hline$p$-Mentha-1(7),8-diene & 1004 & 1003 & 0.1 & $\mathrm{t}$ & 1,2 \\
\hline$(2 E, 4 E)$-Heptadienal & 1005 & 1005 & $\mathrm{t}$ & nd & 1,2 \\
\hline$\delta$-3-Carene & 1009 & 1008 & nd & $\mathrm{t}$ & 1,2 \\
\hline$\alpha$-Terpinene & 1014 & 1014 & 0.2 & $\mathrm{t}$ & 1,2 \\
\hline$p$-Cymene & 1024 & 1020 & 0.9 & 0.1 & 1,2 \\
\hline Limonene & 1023 & 1024 & 0.4 & 0.1 & $1,2,3$ \\
\hline$\beta$-Phellandrene & 1026 & 1025 & 0.1 & $\mathrm{t}$ & 1,2 \\
\hline 1,8-Cineole & 1028 & 1026 & 0.1 & $\mathrm{t}$ & $1,2,3$ \\
\hline (Z)- $\beta$-Ocimene & 1037 & 1032 & $\mathrm{t}$ & $\mathrm{t}$ & 1,2 \\
\hline (E)- $\beta$-Ocimene & 1044 & 1044 & 0.9 & $\mathrm{t}$ & 1,2 \\
\hline Pentyl isobutanoate & 1053 & 1049 & $\mathrm{t}$ & nd & 1,2 \\
\hline$\gamma$-Terpinene & 1057 & 1054 & 0.5 & nd & 1,2 \\
\hline (2E)-Octen-1-ol & 1059 & 1060 & $\mathrm{t}$ & nd & 1,2 \\
\hline
\end{tabular}


TABLE 1 (Continued)

\begin{tabular}{|c|c|c|c|c|c|}
\hline Compound $^{\mathrm{a}}$ & R.I. ${ }^{b}$ & R.I. $^{c}$ & Leaf & Fruit & Identification Method $^{\mathrm{d}}$ \\
\hline Octan-1-ol & 1065 & 1063 & 0.1 & nd & 1,2 \\
\hline trans-Arbusculone & 1066 & 1066 & nd & $\mathrm{t}$ & 1,2 \\
\hline cis-Linalool oxide (furanoid) & 1067 & 1067 & nd & 0.1 & 1,2 \\
\hline trans-Linalool oxide (furanoid) & 1081 & 1084 & nd & 0.1 & 1,2 \\
\hline$m$-Cymenene & 1084 & 1082 & nd & 0.1 & 1,2 \\
\hline Terpinolene & 1086 & 1086 & 3.0 & nd & 1,2 \\
\hline$p$-Cymenene & 1091 & 1089 & 0.1 & nd & 1,2 \\
\hline Linalool & 1098 & 1095 & 0.8 & 0.6 & $1,2,3$ \\
\hline trans-Sabinene hydrate & 1099 & 1098 & nd & 0.1 & 1,2 \\
\hline (E)-6-Methyl-3,5-heptadien-2-one & 1104 & $1100^{\mathrm{e}}$ & $\mathrm{t}$ & nd & 1,2 \\
\hline Nonanal & 1105 & 1100 & $\mathrm{t}$ & nd & 1,2 \\
\hline 1,3,8-p-Menthatriene & 1112 & 1108 & 0.1 & nd & 1,2 \\
\hline cis-p-Menth-2-en-1-ol & 1118 & 1118 & 0.1 & $\mathrm{t}$ & 1,2 \\
\hline Octyl formate & 1127 & 1127 & nd & 0.1 & 1,2 \\
\hline (Z)-Myroxide & 1133 & 1131 & nd & $\mathrm{t}$ & 1,2 \\
\hline cis-p-Mentha-2,8-dien-1-ol & 1134 & 1133 & 0.1 & nd & 1,2 \\
\hline (E)-Myroxide & 1142 & 1140 & $\mathrm{t}$ & 0.1 & 1,2 \\
\hline$\beta$-Pinene oxide & 1153 & 1154 & nd & $\mathrm{t}$ & 1,2 \\
\hline$(2 E)$-Nonenal & 1156 & 1157 & $\mathrm{t}$ & nd & 1,2 \\
\hline Octanoic acid & 1167 & 1167 & nd & $\mathrm{t}$ & 1,2 \\
\hline Ethyl benzoate & 1168 & 1169 & nd & $\mathrm{t}$ & 1,2 \\
\hline cis-Pinocamphone & 1170 & 1172 & 0.1 & nd & 1,2 \\
\hline Terpinen-4-ol & 1179 & 1174 & 1.0 & 0.3 & 1,2 \\
\hline$p$-Cymen-8-ol & 1184 & 1179 & 0.4 & 0.3 & 1,2 \\
\hline Methyl salicylate & 1189 & 1190 & $\mathrm{t}$ & $\mathrm{t}$ & 1,2 \\
\hline Hexyl butanoate & 1192 & 1191 & $\mathrm{t}$ & nd & 1,2 \\
\hline$\alpha$-Terpineol & 1196 & $1198^{\mathrm{f}}$ & 0.4 & 0.1 & 1,2 \\
\hline cis-Sabinol & 1201 & $1202^{\mathrm{g}}$ & $\mathrm{t}$ & $\mathrm{t}$ & 1,2 \\
\hline Decanal & 1204 & 1201 & $\mathrm{t}$ & nd & $1,2,3$ \\
\hline Octyl acetate & 1209 & 1211 & 0.1 & $\mathrm{t}$ & 1,2 \\
\hline$(2 E, 4 E)$-Nonadienal & 1215 & 1210 & $\mathrm{t}$ & nd & 1,2 \\
\hline Nerol & 1223 & 1227 & $\mathrm{t}$ & nd & 1,2 \\
\hline Neral & 1237 & 1235 & $\mathrm{t}$ & $\mathrm{t}$ & 1,2 \\
\hline Geraniol & 1247 & 1249 & $\mathrm{t}$ & $\mathrm{t}$ & 1,2 \\
\hline 2-Phenyl ethyl acetate & 1249 & 1254 & $\mathrm{t}$ & nd & 1,2 \\
\hline Methyl citronellate & 1254 & 1257 & $\mathrm{t}$ & nd & 1,2 \\
\hline (2E)-Decenal & 1257 & 1260 & 0.1 & nd & 1,2 \\
\hline Geranial & 1268 & 1264 & 0.1 & nd & 1,2 \\
\hline iso-3-Thujanyl acetate & 1269 & 1267 & nd & 0.1 & 1,2 \\
\hline Safrole & 1280 & 1285 & 0.1 & $\mathrm{t}$ & 1,2 \\
\hline Thymol & 1290 & 1289 & 0.1 & $\mathrm{t}$ & $1,2,3$ \\
\hline Carvacrol & 1296 & 1298 & 0.1 & nd & 1,2 \\
\hline Eugenol & 1361 & 1356 & 72.8 & 74.7 & $1,2,3$ \\
\hline$\beta$-Elemene & 1389 & 1389 & 0.2 & 0.2 & 1,2 \\
\hline Vanillin & 1398 & 1393 & nd & 0.7 & 1,2 \\
\hline$\beta$-Caryophyllene & 1420 & 1417 & 8.2 & 0.2 & $1,2,3$ \\
\hline Carvone hydrate & 1425 & 1422 & nd & $\mathrm{t}$ & 1,2 \\
\hline
\end{tabular}


TABLE 1 (Continued)

\begin{tabular}{|c|c|c|c|c|c|}
\hline Compound $^{\mathrm{a}}$ & R.I. ${ }^{b}$ & R.I. ${ }^{\mathrm{c}}$ & Leaf & Fruit & Identification Method $^{\mathrm{d}}$ \\
\hline$\beta$-Copaene & 1429 & 1430 & $\mathrm{t}$ & nd & 1,2 \\
\hline$\alpha$-Humulene & 1452 & 1452 & nd & 0.2 & 1,2 \\
\hline$(E)$-Isoeugenol & 1453 & 1448 & nd & $\mathrm{t}$ & 1,2 \\
\hline Sesquisabinene & 1463 & 1457 & nd & 0.1 & 1,2 \\
\hline$\beta$-Chamigrene & 1472 & 1476 & 0.3 & 0.1 & 1,2 \\
\hline$\gamma$-Muurolene & 1475 & 1478 & $\mathrm{t}$ & nd & 1,2 \\
\hline$\alpha$-Amorphene & 1480 & 1483 & $\mathrm{t}$ & nd & 1,2 \\
\hline Germacrene D & 1483 & 1484 & $\mathrm{t}$ & nd & 1,2 \\
\hline$\beta$-Selinene & 1487 & 1489 & 0.7 & 0.4 & 1,2 \\
\hline cis-Cadina-1,4-diene & 1495 & 1495 & $\mathrm{t}$ & nd & 1,2 \\
\hline$\alpha$-Selinene & 1497 & 1498 & 0.8 & 0.5 & 1,2 \\
\hline$\alpha$-Muurolene & 1500 & 1500 & $\mathrm{t}$ & $\mathrm{t}$ & 1,2 \\
\hline$\beta$-Bisabolene & 1505 & 1505 & nd & $\mathrm{t}$ & 1,2 \\
\hline$\delta$-Amorphene & 1508 & 1511 & $\mathrm{t}$ & $\mathrm{t}$ & 1,2 \\
\hline$\gamma$-Cadinene & 1512 & 1513 & $\mathrm{t}$ & nd & 1,2 \\
\hline$(Z)-\gamma$-Bisabolene & 1513 & 1514 & $\mathrm{t}$ & nd & 1,2 \\
\hline$\delta$-Cadinene & 1517 & 1522 & 0.2 & nd & 1,2 \\
\hline cis-Calamenene & 1525 & 1528 & $\mathrm{t}$ & nd & 1,2 \\
\hline Zonarene & 1531 & 1528 & $\mathrm{t}$ & nd & 1,2 \\
\hline$(Z)$-Nerolidol & 1536 & 1531 & $\mathrm{t}$ & nd & 1,2 \\
\hline$\alpha$-Cadinene & 1540 & 1537 & $\mathrm{t}$ & nd & 1,2 \\
\hline$\alpha$-Calacorene & 1545 & 1544 & $\mathrm{t}$ & nd & 1,2 \\
\hline (E)-Nerolidol & 1558 & 1561 & $\mathrm{t}$ & nd & 1,2 \\
\hline Caryophyllenyl alcohol & 1575 & 1570 & 0.1 & nd & 1,2 \\
\hline Caryophyllene oxide & 1582 & 1582 & 0.4 & 3.3 & 1,2 \\
\hline Humulene epoxide II & 1610 & 1608 & 0.1 & 0.8 & 1,2 \\
\hline Junenol & 1622 & 1618 & $\mathrm{t}$ & nd & 1,2 \\
\hline 1-epi-Cubenol & 1628 & 1627 & $\mathrm{t}$ & nd & 1,2 \\
\hline$\gamma$-Eudesmol & 1632 & 1630 & 0.1 & nd & 1,2 \\
\hline Caryophylla-4(12),8(13)-dien-5 $\beta$-ol & 1638 & 1639 & 0.1 & 0.1 & 1,2 \\
\hline epi- $\alpha$-Cadinol & 1638 & 1638 & $\mathrm{t}$ & nd & 1,2 \\
\hline epi- $\alpha$-Muurolol & 1643 & 1640 & 0.1 & nd & 1,2 \\
\hline$\alpha$-Muurolol & 1644 & 1644 & $\mathrm{t}$ & nd & 1,2 \\
\hline Cubenol & 1645 & 1645 & nd & 0.1 & 1,2 \\
\hline$\beta$-Eudesmol & 1449 & 1649 & 0.1 & nd & 1,2 \\
\hline$\alpha$-Cadinol & 1653 & 1652 & nd & 0.1 & 1,2 \\
\hline Selin-11-en-4 $\alpha$-ol & 1657 & 1658 & 0.7 & 0.5 & 1,2 \\
\hline Intermedeol & 1666 & 1665 & $\mathrm{t}$ & nd & 1,2 \\
\hline 14-Hydroxy-9-epi-(E)-caryophyllene & 1670 & 1668 & 0.1 & 0.6 & 1,2 \\
\hline$(Z)$ - $\alpha$-Santalol & 1679 & 1674 & $\mathrm{t}$ & nd & 1,2 \\
\hline$(2 E, 6 Z)$-Farnesal & 1709 & 1713 & $\mathrm{t}$ & nd & 1,2 \\
\hline$(2 E, 6 Z)$-Farnesol & 1714 & 1714 & $\mathrm{t}$ & nd & 1,2 \\
\hline Coniferaldehyde & 1729 & 1728 & $\mathrm{t}$ & 0.3 & 1,2 \\
\hline (E)-Coniferyl alcohol & 1730 & 1733 & nd & 0.2 & 1,2 \\
\hline$(2 E, 6 E)$-Farnesal & 1743 & 1740 & $\mathrm{t}$ & nd & 1,2 \\
\hline $6,10,14$-Trimethyl-2-pentadecanone & 1846 & $1848^{\mathrm{h}}$ & $\mathrm{t}$ & nd & 1,2 \\
\hline
\end{tabular}


TABLE 1 (Continued)

\begin{tabular}{|c|c|c|c|c|c|}
\hline Compound $^{\mathrm{a}}$ & R.I. ${ }^{b}$ & R.I. ${ }^{\mathrm{c}}$ & Leaf & Fruit & 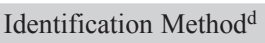 \\
\hline Compound classes & & & $\%$ & $\%$ & \\
\hline Monoterpene hydrocarbons & & & 7.8 & 1.1 & \\
\hline Oxigenated monoterpenes & & & 3.3 & 1.8 & \\
\hline Sesquiterpene hydrocarbons & & & 10.4 & 1.7 & \\
\hline Oxigenated sesquiterpenes & & & 1.8 & 5.5 & \\
\hline Aromatics & & & 72.9 & 75.9 & \\
\hline Others & & & 0.6 & 0.1 & \\
\hline Identified compounds & & & 96.8 & 86.1 & \\
\hline
\end{tabular}

${ }^{\mathrm{a} C}$ Compounds listed in order of elution from poly $(5 \%$ phenyl- $95 \%$ methylsiloxane $)$ column. ${ }^{\mathrm{b}} \mathrm{RI}=$ Experimental retention index relative to a homologous series of $n$-alkanes. ${ }^{\mathrm{c}} \mathrm{RI}=\mathrm{Lit}$.(Adams, 2007). ${ }^{\mathrm{d}}$ Method: $1=$ Retention index on poly $(5 \%$ phenyl-95\% methylsiloxane) column; $2=$ MS spectra; $3=$ Standard; $t=$ traces $(<0.05 \%)$; nd: not detected. ${ }^{\mathrm{e}}($ Pino et al.,

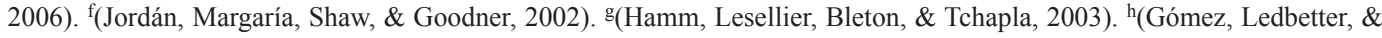
Hartsell, 1993).

P. guatemalensis gave oils which were predominantly aromatic in nature with a terpenic fraction, and several aliphatic and aromatic compounds as trace components.

\section{DISCUSSION}

The leaf oil was rich in the phenylpropanoid compound eugenol (72.8\%) accompanied by a little quantity of safrole $(0.1 \%)$. This oil contained also terpene hydrocarbons (18.2\%) being the main components $\beta$-caryophyllene $(8.2 \%)$ and terpinolene $(3.0 \%)$. The remaining monoterpenes and sesquiterpenes were each one less than $0.9 \%$. Other monoterpenes in the oil were $p$-cymene $(0.9 \%),(E)-\beta$-ocimene $(0.9 \%)$, myrcene $(0.6 \%), \alpha$-phellandrene $(0.6 \%), \gamma$-terpinene $(0.5 \%)$ and limonene $(0.4 \%)$, accompanied by the sesquiterpene hydrocarbons $\alpha$-selinene $(0.8 \%)$ and $\beta$-selinene $(0.7 \%)$. Oxygenated terpenoids were not particularly abundant, with the most prominent members being terpinen-4-ol $(1.0 \%)$, linalool $(0.8 \%)$ and selin-11-en-4- $\alpha$-ol $(0.7 \%)$. The oil of $P$. guatemalensis resembles leaf oils of $P$. dioica from Jamaica with 66.4 to $79.2 \%$ of eugenol (Tucker et al., 1991a). The leaf oil of $P$. dioica from Cuba contained a minor amount of eugenol, 54.3\% (Pino \& Rosado, 1996). One commercial sample of leaf oil from
Jamaica contained $76.0 \%$ of eugenol and $7.1 \%$ of methyl eugenol (Jirovetz et al., 2007), compound not detected in our study nor in the two aforementioned studies on $P$. dioica.

The fruit oil also was rich in eugenol (74.7\%), accompanied by lesser amounts of the benzenoid vanillin $(0.7 \%)$, the phenylpropanoids coniferaldehyde $(0.3 \%)$ and $(E)$ coniferyl alcohol $(0.2 \%)$, together with traces of safrole and $(E)$-isoeugenol. This oil resembles the fruit oil composition of $P$. dioica from Jamaica (Pino, 1999), where the main constituent was eugenol, 68.6-87.0\%. A comparison of our results with those obtained by García Fajardo et al. (1997), from samples of Mexican berries, indicate both qualitative and quantitative differences in the constituents. The Mexican berries contain high quantities of methyl eugenol (48.3-62.7\%) and myrcene $(16.5-17.7 \%)$ and low quantity of eugenol (8.3-17.3\%). The absence of methyl eugenol and the presence of the minor benzenoid and phenylpropanoids above mentioned appears to be a fact that could differentiate the oil of $P$. guatemalensis from other oils of $P$. dioica studied previously. However, to try to chemically differentiate between $P$. guatemalensis and $P$. dioica, it would be necessary to conduct a study with a larger number of wild specimens from various geographic areas of Costa Rica. 


\section{ACKNOWLEDGMENTS}

The authors are grateful to Escuela de Química and Vicerrectoría de Investigación (UCR) for financial support and to L. J. Poveda (Escuela de Ciencias Ambientales, Universidad Nacional) for the species identification.

\section{RESUMEN}

\begin{abstract}
Aceites volátiles de hojas y frutos de Pimenta guatemalensis (Myrtaceae) de Costa Rica. Pimenta es un género de plantas perteneciente a la familia Myrtaceae que contiene cerca de 15 especies, la mayoría ubicadas en las regiones del Caribe del Continente Americano, donde es utilizado con propósitos culinarios y medicinales. Las especies comerciales mejor conocidas son "pimienta de Jamaica" (P. dioica o P. officinalis) y "bay-rum" $(P$. racemosa) y existe muy poca información científica acerca de la especie $P$. guatemalensis. Cuando las hojas y frutos son triturados, desprenden un aroma de composición desconocida. El objetivo del presente estudio fue identificar la composición química de los aceites esenciales de las hojas y frutos de $P$. guatemalensis. La extracción de los aceites esenciales de $P$. guatemalensis, una especie arbórea silvestre en Costa Rica, se efectuó mediante el método de hidrodestilación a presión atmosférica, empleando un aparato de vidrio de tipo Clevenger. Se analizó la composición química de los aceites esenciales mediante cromatografía de gases con detector de ionización de flama (GC/FID) y cromatografía de gases acoplada a un detector de masas (GC/MS) y, utilizando índices de retención en una columna cromatográfica capilar tipo DB-5. En los aceites de hojas se identificaron 103 y en los de frutos 63 compuestos, correspondiendo a $96.8 \%$ y $86.1 \%$, respectivamente, de los constituyentes totales. El aceite de las hojas está constituido principalmente por compuestos de naturaleza fenilpropanoide (72.9\%) y de hidrocarburos monoterpénicos y sesquiterpénicos (18.2\%). Los componentes mayoritarios del aceite de las hojas se identificaron como eugenol $(72.8 \%)$, $\beta$-cariofileno $(8.2 \%)$ y terpinoleno $(3.0 \%)$. El aceite de los frutos está constituido principalmente por eugenol $(74.7 \%)$, monoterpenos y sesquiterpenos oxigenados $(7.3 \%)$ principalmente óxido de cariofileno (3.3\%). Este es el primer informe acerca de la composición química de aceites esenciales obtenidos a partir de esta especie vegetal.
\end{abstract}

Palabras clave: Pimenta guatemalensis, Myrtaceae, aceite esencial, composición química, eugenol, $\beta$-cariofileno, óxido de cariofileno, terpinoleno, Costa Rica.

\section{REFERENCES}

Adams, R. P. (2007). Identification of Essential Oil Components by Gas Chromatography/ Quadrupole Mass
Spectroscopy (4 ${ }^{\text {th }}$ ed.). Carol Stream, Illinois: Allured Publishing Corporation.

Ayedoun, A. M., Adeoti, B. S., Setondji, J., Menut, C., Lamaty, G., \& Bessière, J. M. (1996). Aromatic plants from Tropical West Africa. IV. Chemical composition of leaf oil of Pimenta racemosa (Miller) J. W. Moore var. racemosa from Benin. Journal of Essential Oil Research, 8(2), 207-209.

Barrie, F. R. (2007). Myrtaceae. In B. E. Hammel, M. H. Grayum, C. Herrera, \& N. Zamora (Eds.), Manual de plantas de Costa Rica. Dicotiledóneas. (Haloragaceae-Phytolaccaceae) (p. 772), Vol. 6. (Monographs in systematic botany from the Missouri Botanical Garden, Vol. 111). St. Louis, Missouri: Missouri Botanical Garden Press.

Bello, A., Rodríguez, M. L., Castiñeira, N., Urquiola, A., Rosado, A., \& Pino, J. A. (1995). Chemical composition of the leaf oil of Pimenta racemosa (Mill.) J. Moore from Western Cuba. Journal of Essential Oil Research, 7(4), 423-424.

Bello, A., Urquiola, A., García, J. J., Rosado, A., \& Pino, J. A. (1998). Aceite esencial de las hojas de Pimenta racemosa (Mill.) J. Moore (Myrtaceae) del oeste de Cuba. Ingeniería y Ciencia Química, 18(1), 21-23.

García Fajardo, J., Martínez Sosa, M., Estarrón Espinosa, M., Vilarem, G., Gaset, A., \& Santos, J. M. de. (1997). Comparative study of the oil and supercritical $\mathrm{CO}_{2}$ extract of Mexican pimento (Pimenta dioica Merril). Journal of Essential Oil Research, 9(2), 181-185.

Germosén-Robineau, L. (Ed.). (2005). Farmacopea vegetal caribeña (pp. 336-347). León, Nicaragua: Editorial Universitaria UNAN.

Gómez, E., Ledbetter, C. A., \& Hartsell, P. L. (1993). Volatile compounds in apricot, plum, and their interspecific hybrids. Journal of Agricultural and Food Chemistry, 41(10), 1669-1676.

Hamm, S., Lesellier, E., Bleton, J., \& Tchapla, A. (2003). Optimization of headspace solid microextraction for gas chromatography/mass spectrometry analysis of widely different volatility and polarity terpenoids in olibanum. Journal of Chromatography A, 1018, 73-83.

Jirovetz, L., Buchbauer, G., Stoilova, I., Krastanov, A., Stoyanova, A., \& Schmidt, E. (2007). Spice plants: Chemical composition and antioxidant properties of Pimenta Lindl. essential oils, part 1: Pimenta dioica (L.) Merr. leaf oil from Jamaica. Ernährung/Nutrition, 31(2), 55-62.

Jordán, M. J., Margaría, C. A., Shaw, P. E., \& Goodner, K. L. (2002). Aroma active components in aqueous kiwi fruit essence and kiwi fruit puree by GC-MS and multidimensional GC/GC-O. Journal of Agricultural and Food Chemistry, 50(19), 5386-5390. 
León, J., \& Poveda, L. J. (2000). Los Nombres Comunes de las Plantas en Costa Rica. P. E. Sánchez-Vindas (Ed.), (p. 373). San José, Costa Rica: Editorial Guayacán.

Lim, T. K. (2012). Edible medicinal and non-medicinal plants: Volume 3, Fruits (pp. 655-664). New York: Springer.

Marques, F. A., Wendler, E. P., Baroni, A. C. M., de Oliveira, P. R., Sasaki, B. S., \& Guerrero Jr., P. G. (2010). Leaf essential oil composition of Pimenta pseudocaryophyllus (Gomes) L. R. Landrum native from Brazil. Journal of Essential Oil Research, 22(2), 150-152.

Morton, J. F. (1981). Atlas of Medicinal Plants of Middle America. Bahamas to Yucatan (pp. 628-629). Springfield, Illinois: C.C. Thomas.

Paula, J. A. M., Reis, J. B., Ferreira, L. H. M., Menezes, A. C. S., \& Paula, J. R. (2010). Gênero Pimenta: aspectos botânicos, composição química e potencial farmacológico. Revista Brasileira de Plantas Medicinais, 12(3), 363-379.

Paula, J. A. M., Ferri, P. H., Bara, M. T. F., Tresvenzol, L. M. F., Sá, F. A. S., \& Paula, J. R. (2011). Infraspecific chemical variability in the essential oils of Pimenta pseudocaryophyllus (Gomes) L. R. Landrum (Myrtaceae). Biochemical Systematics and Ecology, 39, 643-650.

Pino, J. A., \& Rosado, A. (1996). Chemical composition of the leaf oil of Pimenta dioica L. from Cuba. Journal of Essential Oil Research, 8(3), 331-332.

Pino, J. A. (1999). Los componentes volátiles de las especias. X. Pimiento. Alimentaria (Madrid), April, 87-90.

Pino, J. A., Bello, A., \& Urquiola, A. (2002). The leaf oil of Pimenta adenoclada (Urb.) Burret from Cuba. Journal of Essential Oil Research, 14(6), 400-401.

Pino, J. A., Marbot, R., Payo, A., Chao, D., \& Herrera. P. (2006). Aromatic plants from Western Cuba. VIII. Composition of the leaf oils of Psidium wrightii Krug et Urb., Lantana involucrata L., Cinnamomum montanum (Sw.) Berchtold et J. Persl. and Caesalpinia violaceae (Mill.) Standley. Journal of Essential Oil Research, 18(2), 170-174.
Pragadheesh, V. S., Yadav, A., Singh, S. C., Gupta, N., \& Chanotiya, C. S. (2013). Leaf essential oil of cultivated Pimenta racemosa (Mill.) J. W. Moore from North India: Distribution of phenylpropanoids and chiral terpenoids. Medicinal \& Aromatic Plants, 2(1): 118. http://dx.doi.org/10.4172/2167-0412.1000118

Rao, P. S., Navinchandra, S., \& Jayaveera, K. N. (2012). An important spice, Pimenta dioica (Linn.) Merill: A review. International Current Pharmaceutical Journal, 1(8), 221-225.

Tucker, A. O., Maciarello, M. J., \& Landrum, L. R. (1991a). Volatile leaf oils of Caribbean Myrtaceae. II. Pimenta dioica (L.) Merr. of Jamaica. Journal of Essential Oil Research, 3(3), 195-196.

Tucker, A. O., Maciarello, M. J., Adams, R. P., Landrum, L. R., \& Zanoni, T. A. (1991b). Volatile leaf oils of Caribbean Myrtaceae. I. Three varieties of Pimenta racemosa (Miller) J. Moore of the Dominican Republic and the commercial bay oil. Journal of Essential Oil Research, 3(5), 323-329.

Tucker, A. O., Maciarello, M. J., Adams, R. P., Landrum, L. R., \& Zanoni, T. A. (1991c). Volatile leaf oils of Caribbean Myrtaceae. III. Pimenta haitiensis (Urban) Landrum of the Dominican Republic. Journal of Essential Oil Research, 3(6), 471-473.

Tucker, A. O., Maciarello, M. J., \& Landrum, L. R. (1992a). Volatile leaf oils of Caribbean Myrtaceae. IV. Pimenta jamaicensis (Britton \& Harris) Proctor of Jamaica. Journal of Essential Oil Research, 4(1), 93-94.

Tucker, A. O., Maciarello, M. J., \&. Landrum, L. R. (1992b). Volatile leaf oils of Caribbean Myrtaceae. V. Pimenta obscura Proctor of Jamaica. Journal of Essential Oil Research, 4(2), 195-196.

van den Dool, H., \& Kratz, P. D. (1963). A generalization of the retention index system including linear temperature programmed gas-liquid partition chromatography, Journal of Chromatography A, 11, 463-471.

Wilson, P. G. (2011). Myrtaceae. In K. Kubitzki (Ed.), The Families and Genera of Vascular Plants. Flowering Plants. Eudicots, Vol. 10 (p. 212). Berlin: Springer. 
